\title{
Clinico-Angiographic Profile and Prevalence of Restenosis in Patients Undergoing Percutaneous Transluminal Coronary Angioplasty to Left Main Coronary Artery: An Observational Cohort Study
}

\author{
Dolly Mathew*, C. G. Sajeev \\ Department of Cardiology, Medical College Calicut, Kerala, India \\ Email: *drdollymathew@gmail.com, sajeev.cg@gmail.com
}

How to cite this paper: Mathew, D. and Sajeev, C.G. (2017) Clinico-Angiographic Profile and Prevalence of Restenosis in Patients Undergoing Percutaneous Transluminal Coronary Angioplasty to Left Main Coronary Artery: An Observational Cohort Study. World Journal of Cardiovascular Diseases, 7, 413-422.

https://doi.org/10.4236/wjcd.2017.711039

Received: October 3, 2017

Accepted: November 27, 2017

Published: November 30, 2017

Copyright $(9) 2017$ by authors and Scientific Research Publishing Inc. This work is licensed under the Creative Commons Attribution International License (CC BY 4.0).

http://creativecommons.org/licenses/by/4.0/

\begin{abstract}
Background: Patients who underwent percutaneous coronary intervention in left main coronary artery (LMCA) requires special concern, being high risk and increasing patient population. The aim of this study was to assess the clinical profile, angiographic status, and prevalence of restenosis in patients who underwent percutaneous transluminal coronary angioplasty (PTCA) in LMCA. Materials and Methods: This observational cohort study included 17 patients who underwent PTCA in LMCA during one-year study period at tertiary care centers in Government Medical College, Kozhikode, India. Data including various risk factors, clinical and angiographic details, stent used, procedural complications and outcomes including rate of restenosis were analyzed. Results: A total of 17 patients (mean age $53.88 \pm 9.80$ years) with $76.47 \%$ of males were included in the study. Smoking and hypertension were the most common risk factors presented in $52.94 \%$ and $47.06 \%$ of patients respectively. Single vessel disease (SVD) of LMCA was the most common pattern observed in $47.10 \%$; the rate of restenosis was observed in $11.76 \%$ patients. Revascularization was performed in one patient (5.88\%) with coronary artery bypass graft and in one patient (5.88\%) with PTCA using drug eluting stent (DES). The overall procedural success was $88.24 \%$ in this study. Survival rate was $100 \%$ at one-year follow-up period. Conclusion: Our study involved patients who underwent PTCA in LMCA, showed smoking as a most prevalent risk factor for coronary artery disease and SVD as a most common pattern, comparatively low rate of restenosis and $100 \%$ of survival rate at one-year follow-up period.
\end{abstract}




\section{Keywords}

Coronary Artery Disease, Drug Eluting Stent, In-Stent Restenosis, Left Main

Coronary Artery, Percutaneous Transluminal Coronary Angioplasty

\section{Introduction}

An epidemiological transition leads to increasing mortality from coronary artery disease (CAD) in developing countries, in contrast to developed countries [1]. In a socioeconomically diverse country like India, a preponderance of CAD is about $7.5 \%$ in urban population and $3.7 \%$ in rural population, because of the genetic predisposition, lifestyle and environmental risk factors [2]. Percutaneous revascularization by percutaneous transluminal coronary angioplasty (PTCA) and stenting are widely practiced among the CAD patients. Since its clinical introduction, PTCA has become an attractive therapeutic option for selected patients, but the problem of restenosis persists. The pace of clinical restenosis had decreased below $5 \%$ for most of the lesion types, with the advent of drug eluting stents (DES) in mid-2003 [3]. However, this low rate of restenosis could not be negligible as the population treated with DES is significantly increasing these days. Stenosis of the left main coronary artery (LMCA) is subjected to the alarming prognosis of any CAD subset [4]. Despite the major advances, the results of PTCA in patients with LMCA stenosis have been disappointing which require significant concern to this increasing patient population. The government medical college, Kozhikode, India is a high-volume center for coronary interventions performing more than 1000 surgical procedures per year and well equipped cardiac catheterization laboratory. Despite this landscape, there is severe paucity of information regarding the angiographic profile, rate of restenosis and well-mannered follow-up in patients undergoing PTCA in LMCA so far, even being a tertiary care center.

The objective of this study was to evaluate the clinical profile, angiographic status, and prevalence of restenosis in patients who underwent PTCA in LMCA in government medical college, Kozhikode, India during the specified study period.

\section{Methods}

\subsection{Study Design and Eligibility}

This observational cohort study was carried out at the department of cardiology, Government medical college, Kozhikode, India from 2011 to 2012 for a total period of 12 months. Ethical approval for the study was given by the Institutional Ethics Committee, Government Medical College, Kozhikode. Patients either male or female who underwent PTCA to LMCA during the study period were evaluated for eligibility criteria. For this study, we included patients who met the 
following inclusion criteria: 1) patients either male or female who falls into the age category of 18 - 70 years; and 2) patients who underwent PTCA to LMCA during the study period of 1-year at study centre irrespective of their clinical presentation viz. chronic stable angina, unstable angina (UA), ST-elevation myocardial infarction (STEMI), and non-ST-elevation myocardial infarction (NSTEMI). The exclusion criteria were: 1) patients who have a severe co-morbid illness, chronic kidney disease, severe left ventricular dysfunction, valvular heart disease; 2) non-consenting patient; and 3) patients who haven't completed 6 months post-procedure follow-up. Total 17 patients who completed planned one-year post-procedure follow-up were enrolled in the study after obtaining informed consent form; those who completed at least 6 months follow-up were included. Subject demographics, clinical variables like age, sex, risk factors (Type 2 diabetes mellitus, hypertension, smoking, family history of premature CAD), and left ventricular ejection fraction (LVEF) were observed. Baseline lipid profile during the first hospitalization, electrocardiogram (ECG), echocardiography evaluation and stress test (TMT) were performed during the study. Family history of premature CAD was considered positive if the first order relative had suffered cardiovascular death, myocardial infarction or coronary revascularization before the 55 years of age.

\subsection{Angiographic Analysis}

Coronary angiography (CAG) was performed initially and at the time of follow-up at one-year or even before if clinically indicated. CAG was assessed visually by two operators, and all the anatomic features, lesion characteristics, procedural complications, luminal narrowing and final results were reviewed. CAG was performed using cath lab system Phillips Allura Xper FD10 at 15 fps. Pre-PTCA angiogram and post-PTCA angiogram of patients were reviewed and compared with the follow-up angiogram.

The SYNTAX scores were calculated by reviewing pre-PTCA angiogram of the patients, and it is subsequently correlated with the clinical profile of the patients. During the study, stent size, pre and post dilatation, any complication during the procedure, cardiac biomarker (troponin I test), stent used for other lesions, TIMI flow, and drugs used were observed. The percentage luminal narrowing within the stent or adjacent to the stent was analyzed. MACE was considered as primary end point.

\subsection{Definitions}

In-stent restenosis (ISR) was defined clinically and angiographically. Clinical ISR was defined as the presentation of recurrent angina or objective evidence of myocardial ischemia. Angiographic ISR was defined as the presence of $>50 \%$ diameter stenosis in the stented segment. ISR had been classified based on the length of the lesion, as focal (lesion $<10 \mathrm{~mm}$ in length) or diffuse (lesion $>10$ $\mathrm{mm}$ in length). In-segment restenosis was defined as restenosis immediately ad- 
jacent to the stented segment, within $5 \mathrm{~mm}$. Acute myocardial infarction (MI) was diagnosed on the basis of prolonged chest pain, electrocardiographic changes and/or a rise in creatine kinase-MB (CKMB) levels more than two times from the normal level. New development of pathological $Q$ waves in two contiguous leads was defined as $\mathrm{Q}$ wave MI; and in the absence of pathological Q waves, an elevation in CKMB level to more than two times from the upper normal limit was defined as non-Q wave MI. Target vessel revascularization (TVR) was defined as any repeated PCI or coronary artery bypass grafting (CABG) to treat luminal narrowing within the stent or within $5 \mathrm{~mm}$ borders adjacent to the stent. Angiographic success was defined as a residual stenosis of $<30 \%$ by visual estimation in the presence of TIMI 3 flow. Procedural success was defined as angiographic success obtained in patients with all target lesions (complete revascularization). Clinical success was defined as procedural success without death, acute MI, repeat revascularization during hospitalization. Major adverse cardiac events (MACE) were defined as the occurrence of cardiac death, nonfatal MI and TVR during hospitalization and follow up period.

\subsection{Statistical Analysis}

All continuous variables were expressed as mean \pm standard deviation (SD), and categorical variables were reported as frequency and percentages.

\section{Results}

Among 23 patients who underwent PTCA in LMCA, 17 patients were studied according to the eligibility criteria over a one-year study period.

\subsection{Patient Characteristics and Clinical Presentation}

The demographics and clinical profile of the study patients are described in Table 1 . The mean age of the study group was $53.88 \pm 9.80$ years. Among the 17 patients studied, 13 patients were males (76.47\%). The incidence of CAD in female (50.25 \pm 11.44 years) occurred at lower age as compared to male (55.00 \pm 9.46 years).

Smoking and hypertension were the most common risk factors presented in $52.94 \%$ and $47.06 \%$ patients respectively. Type 2 diabetes mellitus and family history of CAD at any age, were presented in $29.41 \%$ and $11.76 \%$ patients respectively. Two young females (age $>45$ years) did not have any risk factors. Chronic stable angina was presented in $29.41 \%$ patients followed by UA (23.53\%), STEMI (23.53\%) and NSTEMI (23.53\%). Some degree of overlap was found in patient, viz., patients with chronic stable angina presented later as acute coronary syndrome. ECG changes noted were ST-T change, and QRS complex which matched with current presentations. Among 17 patients, 4 (23.53\%) patients with NSTEMI had positive troponin I test. Three (17.65\%) patients had mild LV dysfunction $(\mathrm{EF}<55)$, and $14(82.35 \%)$ had good LV function (EF $55-85)$. 
Table 1. Demographic and clinical profile of the patients.

\begin{tabular}{|c|c|c|c|c|}
\hline \multirow{2}{*}{ Sr. No. } & \multirow{2}{*}{ Variables, n (\%) } & \multicolumn{3}{|c|}{$\mathrm{N}=17$ patients } \\
\hline & & Male & Female & Total \\
\hline \multirow[t]{5}{*}{1} & Age (years) & & & \\
\hline & $35-45$ & $2(11.76 \%)$ & $2(11.76 \%)$ & $4(23.53 \%)$ \\
\hline & $46-55$ & $3(17.65 \%)$ & $0(0 \%)$ & $3(17.65 \%)$ \\
\hline & $56-65$ & $7(41.18 \%)$ & $2(11.76 \%)$ & $9(52.94 \%)$ \\
\hline & $66-75$ & $1(5.88 \%)$ & $0(0 \%)$ & $1(5.88 \%)$ \\
\hline 2 & Sex distribution & $13(76.47 \%)$ & $4(23.53 \%)$ & $17(100 \%)$ \\
\hline \multirow[t]{7}{*}{3} & Risk factors & & & \\
\hline & Hypertension & $7(53.84 \%)$ & $1(25.00 \%)$ & $8(47.06 \%)$ \\
\hline & Type 2 Diabetes Mellitus & $5(38.46 \%)$ & $0(0 \%)$ & $5(29.41 \%)$ \\
\hline & Family history of CAD at any age & $2(15.38 \%)$ & $0(0 \%)$ & $2(11.76 \%)$ \\
\hline & Previous CAD & $1(7.69 \%)$ & $1(25.00 \%)$ & $2(11.76 \%)$ \\
\hline & Smoking & $9(69.23 \%)$ & $0(0 \%)$ & $9(52.94 \%)$ \\
\hline & Dyslipidemia & $3(23.08 \%)$ & $2(50.00 \%)$ & $5(29.41 \%)$ \\
\hline \multirow[t]{5}{*}{4} & Clinical presentation & & & \\
\hline & Chronic stable angina & $4(30.77 \%)$ & $1(25.00 \%)$ & $5(29.41 \%)$ \\
\hline & Unstable angina (UA) & $3(23.08 \%)$ & $1(25.00 \%)$ & $4(23.53 \%)$ \\
\hline & STEMI & $3(23.08 \%)$ & $1(25.00 \%)$ & $4(23.53 \%)$ \\
\hline & NSTEMI & $3(23.08 \%)$ & $1(25.00 \%)$ & $4(23.53 \%)$ \\
\hline \multirow[t]{3}{*}{5.} & LVEF & & & \\
\hline & EF: $55-85$ & $10(76.92 \%)$ & $4(100 \%)$ & $14(82.35 \%)$ \\
\hline & $\mathrm{EF}<55$ & $3(23.08 \%)$ & $0(0 \%)$ & $3(17.65 \%)$ \\
\hline
\end{tabular}

CAD: Coronary artery disease; STEMI: ST-elevation myocardial infarction; NSTEMI: non-ST-elevation myocardial infarction; LVEF: Left ventricular ejection fraction; EF: ejection fraction.

\subsection{Initial Angiographic Profile}

Initial angiographic profile of the patients describing diseased vessels are shown in Table 2. Initial angiographic analysis revealed that single vessel disease (SVD) involving LMCA was most common pattern observed in 8 (47.10\%) patients. Double vessel disease (DVD) with LMCA and LAD was found in 4 (23.53\%) patients. Triple vessel disease (TVD) with LMCA, LAD, and right coronary artery (RCA) was observed in 3 (17.65\%) patients; LMCA, LCx (Left Circumflex artery) and RCA combination was found in $1(5.88 \%)$ patient, and LMCA, LAD and LCx in 1 (5.88\%) patient. After LCMA, LAD (47.06\%) was the most common vessel involved followed by RCA (23.53\%) and LCx (11.76\%). SYNTAX score was presented less than 28 , which was calculated based on the finding of initial CAG [5]. 
Table 2. Initial angiographic profile of the patients.

\begin{tabular}{ccccc}
\hline \multirow{2}{*}{ Vessel involved } & \multicolumn{4}{c}{ N (\%) } \\
\cline { 2 - 5 } & SVD & DVD & TVD & Total \\
& $\mathbf{8 ( 4 7 . 1 0 \% )}$ & $\mathbf{4 ( 2 3 . 5 3 \% )}$ & $\mathbf{5 ( 2 9 . 4 1 \% )}$ & $\mathbf{1 7 ( 1 0 0 \% )}$ \\
\hline LCMA & $8(100 \%)$ & $4(100 \%)$ & $5(100 \%)$ & $17(100 \%)$ \\
LAD & $0(0 \%)$ & $4(100 \%)$ & $4(80.00 \%)$ & $8(47.06 \%)$ \\
RCA & $0(0 \%)$ & $0(0 \%)$ & $4(80.00 \%)$ & $4(23.53 \%)$ \\
LCx & $0(0 \%)$ & $0(0 \%)$ & $2(40.00 \%)$ & $2(11.76 \%)$ \\
\hline
\end{tabular}

SVD: Single vessel disease; LMCA: Left main coronary angiography; DVD: Double vessel disease; LAD: Left anterior descending; RCA: Right coronary artery; LCx: Left circumflex artery.

\subsection{Coronary Interventions}

DESs were deployed for all LMCA lesions. Diameter and length of the stent varied from $3-4 \mathrm{~mm}$ and $8-24 \mathrm{~mm}$ respectively. Total 19 stents were used to treat 17 LMCA lesions, of which 17 were sirolimus eluting stents and 2 were biolimus eluting stents. Apart from the index LMCA lesions, 9 other lesions were stented in vessels other than LMCA. Among these 9 lesions, 2 lesions were stented with bare metal stents (BMSs), and seven lesions were with DESs. All patients had achieved TIMI III flow in all the lesions post-procedural. During the index procedure one patient had slow flow and one had no-reflow phenomenon. Pre-dilatation with balloon was done for $14(82.35 \%)$ patients with an average pressure of $12.14 \mathrm{~atm}$ before the stent implantation and only 2 patients needed post dilatation at $20 \mathrm{~atm}$ after stenting. The most commonly prescribed drugs for such patients were dual anti-platelet (100\%), statins (100\%), nitrates (100\%) and angiotensin converting enzymes inhibitors (94.12\%). Additional drugs used were beta blocker $(76.12 \%)$ and proton pump inhibitors used whenever necessary.

\subsection{Follow-Up}

Follow-up CAG was performed in all $17(100 \%)$ patients with average follow-up period of 10.5 months. Table 3 shows the results of angiographic and clinical follow ups. Out of 17 patients, 12 (70.59\%) patients did not have any flow limiting lesions in coronary arteries. Two (11.76\%) patients developed a significant degree of ISR in LMCA. Among these two patients, one (5.88\%) patient presented total ISR and other one (5.88\%) patient presented 70\% ISR of LMCA and proximal LAD. TVR after follow up CAG was performed for both the patients having ISR in LMCA. CABG with left internal mammary artery (LIMA) in LAD graft was performed in one patient (5.88\%) having $100 \%$ ISR in LMCA. PTCA with DES was performed in one other patient (5.88\%) with 70\% ISR in LMCA and proximal LAD. In-segment restenosis involving $60 \%$ proximal LAD was observed in one patient $(5.88 \%)$, but inducible ischemia was not found on stress test (TMT). One (5.88\%) patients had $60 \%$ ISR of LAD; one (5.88\%) patients had $50 \%$ ISR of RCA, but the test for inducible ischemia was found negative for both. 
Table 3. Angiographic and clinical data follow-up.

\begin{tabular}{cc}
\hline Variables & $\mathbf{N}(\%)$ \\
Follow-up & $17(100 \%)$ \\
In-stent restenosis in LMCA stent & $2(11.76 \%)$ \\
In-stent restenosis in LAD stent & $1(5.88 \%)$ \\
In-stent restenosis in RCA stent & $1(5.88 \%)$ \\
In-segment restenosis & $1(5.88 \%)$ \\
Target Vessel Revascularization (TVR) & $2(11.76 \%)$ \\
PTCA & $1(5.88 \%)$ \\
Angina & $1(5.88 \%)$ \\
Dyspnoea & $7(41.17 \%)$ \\
Procedural success & $5(29.41 \%)$ \\
Survival rate at one year & $15(88.24 \%)$ \\
\hline
\end{tabular}

LMCA: Left main coronary artery; LAD: left anterior descending; RCA: right coronary artery; PTCA: percutaneous transluminal coronary angioplasty; CABG: coronary artery bypass grafting.

Symptomatology during follow-up showed dyspnea in 5 (29.41\%) patients, angina in $7(41.18 \%)$ patients with a variable degree of overlap of these symptoms; 7 (41.18\%) patients were symptom-free. Electrocardiogram of one patient showed evolved anterior wall myocardial infarction in the follow-up ECG. Echo evaluation was performed at one-month post procedure where regional wall motion abnormality was observed in $6(35.29 \%)$ patients. From the three patients who had LV dysfunction, two patients had been improved LV function after surgery, and one had persisting documented LV dysfunction. TMT after the follow-up CAG was performed to assess the intermediate lesion in LAD for two patients; both were negative.

No death in relation to procedure or related to complications during the study period was observed.

\section{Discussion}

The major findings of this study were: The patient population undergoing PTCA in LCMA at our institute was between the age group of $35-67$ years (mean age $53.88 \pm 9.80 \%)$. Smoking and hypertension were the most common risk factor observed in $52.94 \%$ and $47.06 \%$ population respectively. SVD was the most common angiographic pattern (47.10\%) observed. The rate of ISR in LMCA was significant in two patients (11.76\%). The rate TVR was $11.76 \%$; revascularization was achieved with CABG in one patient and PTCA with DES in one patient. The overall procedural success was $88.24 \%$ in our study. Survival rate was $100 \%$ at one year follow-up period.

Since the first clinical picture of LMCA disease by Herrick in 1912 [6], numerous studies have shown that stenosis of the left main coronary artery is of 
vital prognostic importance. There is an extensive myocardial area at risk while operating the LMCA disease which appends special management problems during revascularization [7]. With the increasing number of DES-treated patients, the prevalence of ISR is becoming an arena of concern. Our hospital is performing more than one thousand cases of PTCA per year, so an even lower rate of restenosis becomes significantly important. The present study provides an insight into the clinical and angiographic profile of the patients who underwent PTCA in LMCA and the prevalence of restenosis.

A total of 17 patients were enrolled in the study according to the inclusion and exclusion criteria. According to the INTERHEART study [8], the median age for the presentation of CAD in the South Asian population is 53 years which was consistent with our study but earlier than the western population as feature in COURAGE trial (62 years) [9]. In contrary to the Beig et al. study [10], CAD was presented earlier in female (mean age $50.25 \pm 11.44$ ) compared to men (mean age 55.00 \pm 9.46$)$ in our study. Sex ratio observed was 3.25:1 (76.47\% male) and this can be attributed to the gender bias which is also a similar feature in INTERHEART study $(75.9 \%$ male) [11]. The most common risk factor noted was past or current smoking in $52.94 \%$ patients which is predominant in the male. Smoking is also the most prevalent risk factor in the Beig et al. study [10], and Sharma et al. study [2]. The prevalence of hypertensive patients was $47.06 \%$ which was quite higher than the prevalence seen in the INTERHEART study (39.0\%) [11] and De Gregorio et al. study (44\% for $<75$ years cohort) [12]. Type 2 Diabetes Mellitus was present in $29.41 \%$ patients which were distinctly more than the other observed study [11] [12] [13]. Two young females (age $<45$ years) did not observe with any risk factors. Whether or not this displays a picture of risk factors of the genesis of CAD in our population, it needs to be ascertained in larger epidemiological studies. The clinical presentations showed $29.41 \%$ patients presented with chronic stable angina and together the acute coronary syndrome constituted $70.59 \%$ was the most common presentation included UA, STEMI, and USTEMI similar to Sharma et al. study [2]. SVD was the most common angiographic pattern, followed by DVD and TVD observed in Beig et al. [10] and Sharma et al. study [2]. Where in our study, SVD of LMCA was most common pattern observed in $47.10 \%$ patients followed by TVD (29.41\%) and DVD (23.53\%). During index coronary intervention, total 19 stents used to treat 17 LMCA lesions, from which simultaneous kissing stenting (SKS) technique was used for $11.76 \%$ patients with LMCA shaft and distal true bifurcation disease. From these lesions $11.76 \%$ patients developed ISR in the stented segment of LMCA. Vessels other than LMCA were treated with 9 other stents.

Primary end point was achieved in two (11.76\%) patients as restenosis in LMCA. The cumulative percent of ISR in LMCA obtained was $11.76 \%$, from which $5.88 \%$ underwent $C A B G$ with left internal mammary artery (LIMA) to LAD graft, and 5.88\% underwent repeat PTCA in LMCA stent in the present study. A Russian three year follow-up study of 124 patients underwent the high risk of surgery with stenting of unprotected LMCA performed with standard 
metal stents, from August 2002 to November 2004 witness restenosis rate of $20.2 \%$ (20 patients). Repeat PCI was performed in 18 patients in that particular study [14]. Another study by Chieffo et al., a contemporary, nonrandomized, single center examined 249 patients with LMCA disease from which 107 patients were treated with DES and 142 patients were surgically revascularized. Lower occurrence of the MACE was associated with DES treatment, as compared to surgery was reported in Chieffo et al. study. Restenosis rate during the clinical follow-up of 12 months as per this study was reported as 19.6\% [15]. The rate of restenosis appears slightly higher in these studies compared to the present study, but the case number was exceedingly less in our study.

Cardiac death was not occurred in the examined group during hospitalization and follow up period. $100 \%$ survival rate at one year was observed in our study. Total survival of patients in a similar Russian study was $95.2 \%, 91.9 \%$ and $90.3 \%$ in one, two and three years, respectively [14]. A recent meta-analysis, considering results of 16 observational studies on 1278 patients undergoing PCI with DES for unprotected LMCA disease, showed a low in-hospital mortality rate of $2.3 \%$ and a low mid-term mortality rate of $5.5 \%$ at a median of 10 -month follow-up [16]. Long-term follow-up is required to better determine the relative impact of coronary revascularization with DES on late survival. The overall procedural success was $88.24 \%$ in our study. $11.76 \%$ patients exhibited restenosis which was a primary outcome of our study. The only procedural complication faced in our study was that ISR of LCx left untreated as it could not be recrossed in one patient. Survival rate was $100 \%$ at one year in this study.

This study has some major limitations. The primary limitation was a very small sample size of only 17 patients and a truncated study period which became a hindrance for evaluating some important CAD aspects. The skewed presentation of sex ratio limits the comparison between male and female's outcome. Influence of demographics and risk factors on the rate of restenosis could not be studied again due to small sample size. Long term follow up could be an aid in presenting a better picture of restenosis rate survival rates, and any potential MACE.

\section{Conclusion}

In spite of the advent of DES, ISR nonetheless happens, and this represents a technical challenge. Restenosis rate elucidated that PTCA in LMCA was low and rate of survival was favorable at follow-up period. However, it needs to be conducted in a larger population and for a longer duration, as the percutaneous coronary intervention in LMCA is rising steadily.

\section{References}

[1] Gupta, R., Mohan, I. and Narula, J. (2016) Trends in Coronary Heart Disease Epidemiology in India. Annals of global health, 82, 307-315.

https://doi.org/10.1016/j.aogh.2016.04.002

[2] Sharma, R., Bhairappa, S., Prasad, S., et al. (2014) Clinical Characteristics, Angio- 
graphic Profile and in Hospital Mortality in Acute Coronary Syndrome Patients in South Indian Population. Heart India, 2, 65-69.

https://doi.org/10.4103/2321-449X.140228

[3] Baim, D.S., Mauri, L. and Cutlip, D.C. (2006) Drug-Eluting Stenting for Unprotected Left Main Coronary Artery Disease. Journal of the American College of Cardiology, 47, 878-881. https://doi.org/10.1016/j.jacc.2005.12.016

[4] Tommaso, C.L., Vogel, J.H. and Vogel, R.A. (1992) Coronary Angioplasty in High-Risk Patients with Left Main Coronary Stenosis: Results from the National Registry of Elective Supported Angioplasty. Catheterization and Cardiovascular Interventions, 25, 169-173. https://doi.org/10.1002/ccd.1810250302

[5] Syntax Score. http://www.syntaxscore.com/calculator/start.htm

[6] Herrick, J.B. (1912) Clinical Features of Sudden Obstruction of the Coronary Arteries. Journal of the American Medical Association, 59, 2015-2022.

https://doi.org/10.1001/jama.1912.04270120001001

[7] Rawat, S., Mehta, Y., Yaseen, T., et al. (2009) CABG in a Patient Recovering from Dengue Fever. Indian Heart Journal, 61, 386-388.

[8] Yusuf, S., Hawken, S., Ônpuu, S., et al. (2004) Effect of Potentially Modifiable Risk Factors Associated with Myocardial Infarction in 52 Countries (the INTERHEART Study): Case-Control Study. The Lancet, 364, 937-952. https://doi.org/10.1016/S0140-6736(04)17018-9

[9] Cleland, J.G., Coletta, A.P. and Clark, A.L. (2007) Clinical Trials Update from the American College of Cardiology 2007: ALPHA, EVEREST, FUSION II, VALIDD, PARR-2, REMODEL, SPICE, COURAGE, COACH, REMADHE, pro-BNP for the Evaluation of Dyspnoea and THIS-Diet. European Journal of Heart Failure, 9, 740-745. https://doi.org/10.1016/j.ejheart.2007.04.004

[10] Beig, J.R., Shah, T.R., Hafeez, I., et al. (2017) Clinico-Angiographic Profile and Procedural Outcomes in Patients Undergoing Percutaneous Coronary Interventions: The Srinagar Registry. Indian Heart Journal, 69, 589-596. https://doi.org/10.1016/j.ihj.2017.01.021

[11] Smyth, A., O’Donnell, M., Lamelas, P., et al. (2016) Physical Activity and Anger or Emotional Upset as Triggers of Acute Myocardial Infarction. Circulation, 134, 1059-1067. https://doi.org/10.1161/CIRCULATIONAHA.116.023142

[12] De Gregorio, J., Kobayashi, Y., Albiero, R., et al. (1998) Coronary Artery Stenting in the Elderly: Short-Term Outcome and Long-Term Angiographic and Clinical Follow-Up. Journal of the American College of Cardiology, 32, 577-583. https://doi.org/10.1016/S0735-1097(98)00287-3

[13] Ruifrok, W., Jessurun, G., Tio, R., et al. (2005) Angioplasty of the Left Main Coronary Artery: Mid-Term Follow-Up at University Medical Centre Groningen. Netherlands Heart Journal, 13, 349-354.

[14] Batyraliev, T., Fettser, D., Karben, Z., et al. (2008) Stenting of Unprotected Left Main Coronary Artery: Complications during 3 Years Follow Up. Kardiologiia, 49, 22-26.

[15] Chieffo, A., Stankovic, G., Bonizzoni, E., et al. (2005) Early and Mid-Term Results of Drug-Eluting Stent Implantation in Unprotected Left Main. Circulation, 111, 791-795. https://doi.org/10.1161/01.CIR.0000155256.88940.F8

[16] Biondi-Zoccai, G.G., Lotrionte, M., Moretti, C., et al. (2008) A Collaborative Systematic Review and Meta-Analysis on 1278 Patients Undergoing Percutaneous Drug-Eluting Stenting for Unprotected Left Main Coronary Artery Disease. American Heart Journal, 155, 274-283. https://doi.org/10.1016/j.ahj.2007.10.009 